\title{
2005 PRETEXT: a revised staging system for primary malignant liver tumours of childhood developed by the SIOPEL group
}

\author{
Derek J. Roebuck • Daniel Aronson • Philippe Clapuyt • \\ Piotr Czauderna • Jean de Ville de Goyet • \\ Frédéric Gauthier • Gordon MacKinlay • \\ Rudolf Maibach • Kieran McHugh • Øystein E. Olsen • \\ Jean-Bernard Otte • Danièle Pariente • Jack Plaschkes • \\ Margaret Childs $\cdot$ Giorgio Perilongo
}

Received: 16 September 2006 / Accepted: 29 October 2006 / Published online: 21 December 2006

(C) Springer-Verlag 2006

\begin{abstract}
Over the last 15 years, various oncology groups throughout the world have used the PRETEXT system for staging malignant primary liver tumours of childhood. This paper, written by members of the radiology and surgery committees of the International Childhood Liver Tumor Strategy Group (SIOPEL), presents various clarifications and revisions to the original PRETEXT system.
\end{abstract}

Keywords Liver - Tumour · Hepatoblastoma · Staging . Children

D. J. Roebuck $(\varangle) \cdot K$. McHugh $\cdot \varnothing$. E. Olsen

Department of Radiology, Great Ormond Street Hospital,

London WC1N 3JH, UK

e-mail: roebud@gosh.nhs.uk

\section{Aronson}

Academisch Medisch Centrum/Universiteit van Amsterdam, locatie Emma Kinderziekenhuis AMC,

Amsterdam, The Netherlands

P. Clapuyt $\cdot$ J. de Ville de Goyet $\cdot$ J.-B. Otte

Departments of Surgery and Medical Imaging,

Université Catholique de Louvain,

Cliniques Universitaires Saint-Luc,

Brussels, Belgium

P. Czauderna

Department of Pediatric Surgery, Medical University of Gdansk,

Gdansk, Poland

F. Gauthier $\cdot$ D. Pariente

Centre Hospital-Universitaire de Bicêtre APHP,

Le Kremlin-Bicêtre cedex, France

\section{Introduction}

The PRETEXT system was designed by the International Childhood Liver Tumor Strategy Group (SIOPEL) for staging and risk stratification of liver tumours $[1,2]$. PRETEXT is used to describe tumour extent before any therapy, thus allowing more effective comparison between studies conducted by different groups. The system has good interobserver reproducibility [3] and good prognostic value in children with hepatoblastoma [2-5], and is the basis of risk stratification in current SIOPEL hepatoblastoma studies. Most other study groups now use the PRETEXT system

\section{G. MacKinlay}

Department of Surgery,

Royal Hospital for Sick Children,

Edinburgh, UK

R. Maibach

SIAK Coordinating Center,

Bern, Switzerland

J. Plaschkes

Department of Pediatric Surgery, Inselspital,

Bern, Switzerland

\section{Childs}

United Kingdom Children's Cancer Study Group,

Leicester, UK

G. Perilongo

Division of Hematology/Oncology,

Department of Pediatrics, Padua University Hospital,

Padua, Italy 

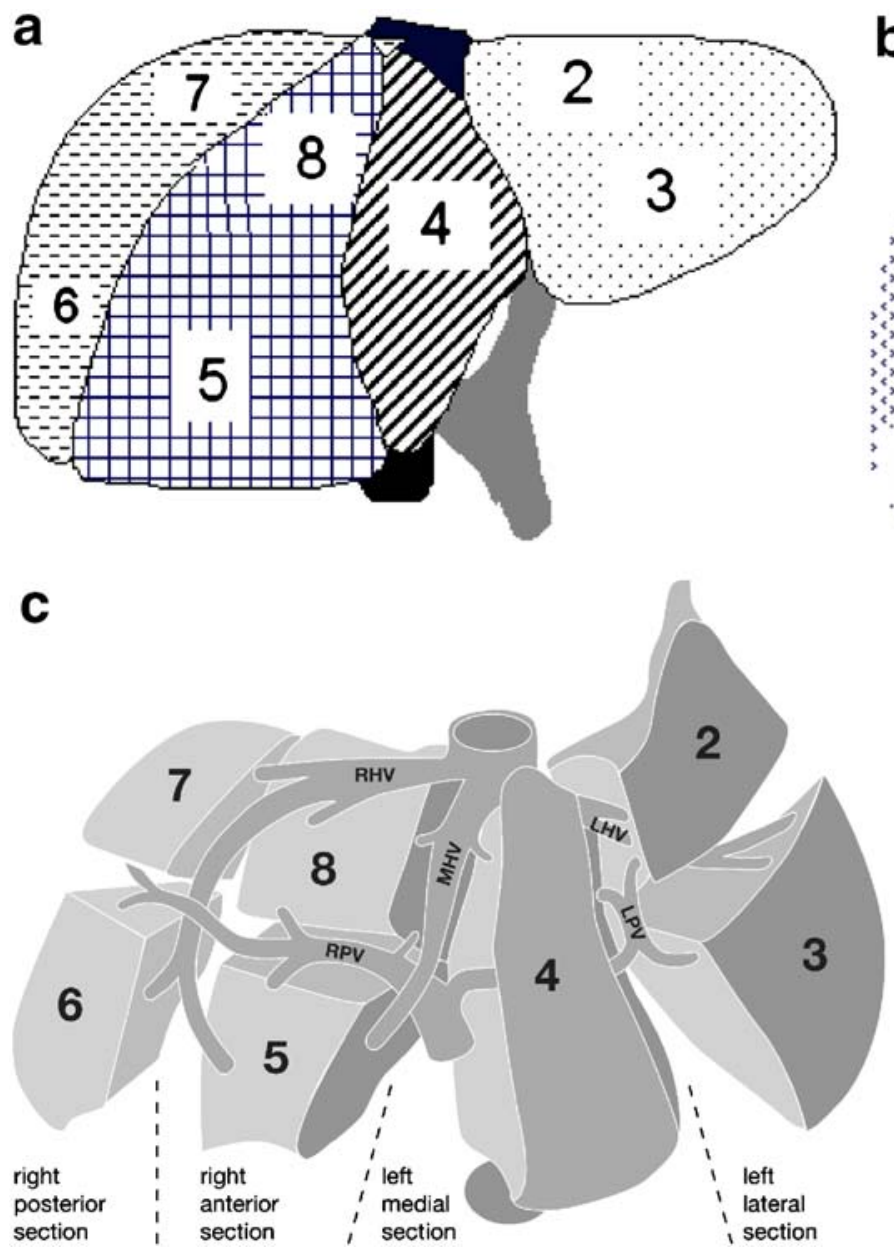

Fig. 1 Schematic representations of the segmental anatomy of the liver. a Frontal view of the liver. The numerals label Couinaud's segments 2 to 8 . b The hepatic veins (black) and the intrahepatic branches of the portal veins (grey) are shown. Segment 1 (equivalent to the caudate lobe) is seen to lie between the portal vein and the inferior vena cava. c Exploded frontal view of the segmental anatomy of the liver. The umbilical portion of the left portal vein $(L P V)$ separates the left medial section from the left lateral section (LLS). Segment 1 is obscured in this view. Note that the term "section" has been used in preference to "segment" or "sector" (see text). d Transverse section of

to describe imaging findings at diagnosis, even if this is not their main staging system.

Certain limitations of the system have become obvious over the last 15 years. In addition, there have been significant advances in imaging during this period [6]. This paper is the report of a working party that met in June 2005 to update the PRETEXT system.

PRETEXT staging is based on Couinaud's system of segmentation of the liver (Fig. 1) [7]. The liver segments are grouped into four sections as follows: segments 2 and 3 (left lateral section), segments $4 \mathrm{a}$ and $4 \mathrm{~b}$ (left medial section), segments 5 and 8 (right anterior section) and segments 6 and 7 (right posterior section). The term section is used (where other authors use segment or sector) to avoid terminological confusion.

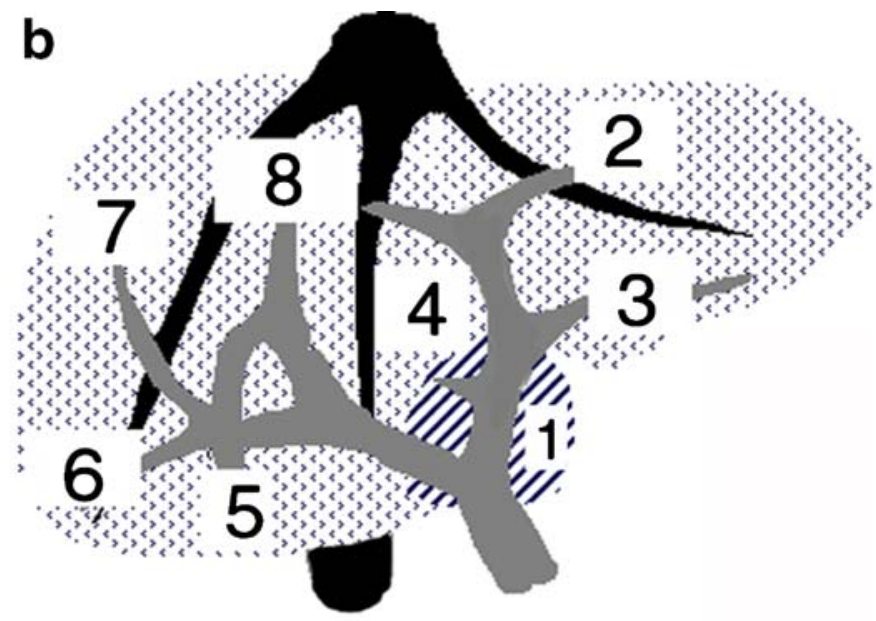

d

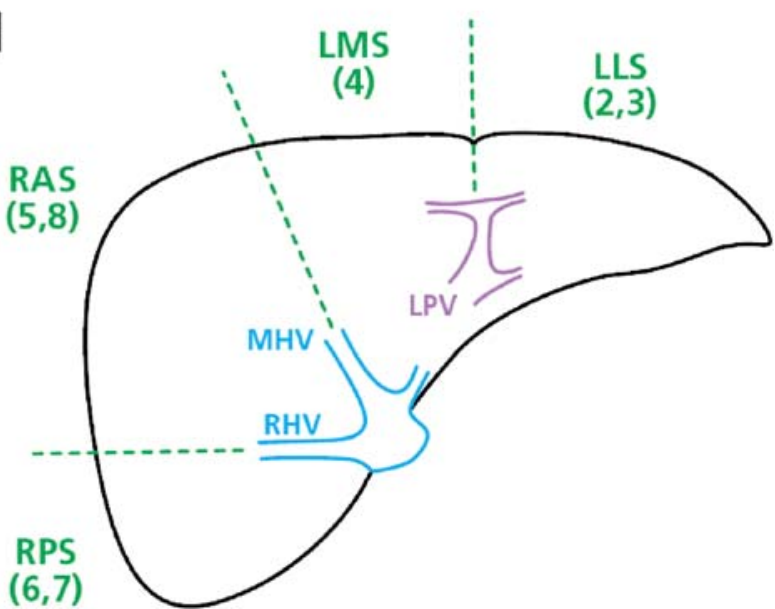

the liver shows the planes of the major venous structures used to determine the PRETEXT number. The hepatic (blue) and portal (purple) veins define the sections of the liver (2-8). This schematic diagram shows how the right hepatic $(R H V)$ and middle hepatic $(M H V)$ veins indicate the borders of the right anterior section $(R A S)$ with the right posterior $(R P S)$ and left medial $(L M S)$ sections. Note that the left portal vein $(L P V)$ actually lies caudal to the confluence of the hepatic veins and is not seen in the same transverse image. The left hepatic vein $(L H V)$ runs between segments 2 and 3 and is not used in PRETEXT staging

In the original system, the caudate lobe (segment 1) was ignored. The PRETEXT number was derived by subtracting the highest number of contiguous liver sections that

Table 1 Definitions of PRETEXT number (see text for PRETEXT number of tumours involving the caudate lobe)

\begin{tabular}{ll}
\hline PRETEXT number & Definition \\
\hline I & One section is involved and three adjoining \\
& sections are free \\
II & One or two sections are involved, \\
& but two adjoining sections are free \\
III & Two or three sections are involved, \\
& and no two adjoining sections are free \\
IV & All four sections are involved
\end{tabular}


Table 22005 PRETEXT staging: additional criteria

\begin{tabular}{|c|c|c|c|c|}
\hline Caudate lobe involvement & $\mathrm{C}$ & $\begin{array}{l}\mathrm{C} 1 \\
\mathrm{C} 0\end{array}$ & $\begin{array}{l}\text { Tumour involving the caudate lobe } \\
\text { All other patients }\end{array}$ & $\begin{array}{l}\text { All } \mathrm{C} 1 \text { patients are at } \\
\text { least PRETEXT II }\end{array}$ \\
\hline \multirow[t]{3}{*}{$\begin{array}{l}\text { Extrahepatic abdominal } \\
\text { disease }\end{array}$} & \multirow[t]{3}{*}{$\mathrm{E}$} & E0 & $\begin{array}{l}\text { No evidence of tumour spread in the abdomen (except } \\
\mathrm{M} \text { or } \mathrm{N} \text { ) }\end{array}$ & \multirow[t]{7}{*}{$\begin{array}{l}\text { Add suffix "a" if ascites } \\
\text { is present, e.g., E0a }\end{array}$} \\
\hline & & E1 & $\begin{array}{l}\text { Direct extension of tumour into adjacent organs or } \\
\text { diaphragm }\end{array}$ & \\
\hline & & E2 & Peritoneal nodules & \\
\hline \multirow[t]{2}{*}{ Tumour focality } & \multirow[t]{2}{*}{$\mathrm{F}$} & F0 & Patient with solitary tumour & \\
\hline & & $\mathrm{F} 1$ & Patient with two or more discrete tumours & \\
\hline \multirow[t]{2}{*}{$\begin{array}{l}\text { Tumour rupture or } \\
\text { intraperitoneal haemorrhage }\end{array}$} & \multirow[t]{2}{*}{$\mathrm{H}$} & $\mathrm{H} 1$ & $\begin{array}{l}\text { Imaging and clinical findings of intraperitoneal } \\
\text { haemorrhage }\end{array}$ & \\
\hline & & $\mathrm{HO}$ & All other patients & \\
\hline \multirow[t]{2}{*}{ Distant metastases } & \multirow[t]{2}{*}{ M } & M0 & No metastases & \multirow{5}{*}{$\begin{array}{l}\text { Add suffix or suffixes to } \\
\text { indicate location (see text) }\end{array}$} \\
\hline & & M1 & Any metastasis (except E and N) & \\
\hline \multirow[t]{3}{*}{ Lymph node metastases } & \multirow[t]{3}{*}{$\mathrm{N}$} & No & No nodal metastases & \\
\hline & & N1 & Abdominal lymph node metastases only & \\
\hline & & $\mathrm{N} 2$ & $\begin{array}{l}\text { Extra-abdominal lymph node metastases (with or without } \\
\text { abdominal lymph node metastases) }\end{array}$ & \\
\hline \multirow[t]{3}{*}{ Portal vein involvement } & \multirow[t]{3}{*}{$\mathrm{P}$} & P0 & $\begin{array}{l}\text { No involvement of the portal vein or its left or right } \\
\text { branches }\end{array}$ & \multirow{3}{*}{$\begin{array}{l}\text { See text for definition of } \\
\text { involvement. Add suffix "a" } \\
\text { if intravascular tumour is } \\
\text { present, e.g., P1a }\end{array}$} \\
\hline & & P1 & $\begin{array}{l}\text { Involvement of either the left or the right branch of the } \\
\text { portal vein }\end{array}$ & \\
\hline & & $\mathrm{P} 2$ & Involvement of the main portal vein & \\
\hline \multirow[t]{4}{*}{$\begin{array}{l}\text { Involvement of the IVC and/ } \\
\text { or hepatic veins }\end{array}$} & \multirow[t]{4}{*}{$\mathrm{V}$} & V0 & $\begin{array}{l}\text { No involvement of the hepatic veins or inferior vena cava } \\
\text { (IVC) }\end{array}$ & \multirow{4}{*}{$\begin{array}{l}\text { See text for definition of } \\
\text { involvement. Add suffix "a" if } \\
\text { intravascular tumour is present, } \\
\text { e.g., V3a }\end{array}$} \\
\hline & & V1 & Involvement of one hepatic vein but not the IVC & \\
\hline & & $\mathrm{V} 2$ & Involvement of two hepatic veins but not the IVC & \\
\hline & & $\mathrm{V} 3$ & Involvement of all three hepatic veins and/or the IVC & \\
\hline
\end{tabular}

were not involved by tumour from four [1]. This number is, very roughly, an estimate of the difficulty of the expected surgical procedure (Table 1). Pedunculated tumours are considered to be confined to the liver and to occupy only the section(s) from which they originate.

In addition to describing the intrahepatic extent of the primary tumour(s), the PRETEXT system includes certain other criteria. These assess involvement of the inferior vena cava (IVC) or hepatic veins (designated V), involvement of the portal veins $(\mathrm{P})$, extrahepatic abdominal disease $(\mathrm{E})$ and distant metastases $(\mathrm{M})$.

The purpose of the 2005 revision was to improve the original definitions of the PRETEXT stages, to clarify the criteria for "extrahepatic" disease, and to add new criteria (Table 2). The term "extrahepatic" disease is confusing, and these categories will in future be called "additional criteria". There is still much to be learned about prognostic factors in the primary malignant liver tumours of childhood. An important goal of these changes, therefore, is to improve our ability to identify prognostic imaging findings, and thereby refine risk stratification.

Although the PRETEXT system is principally used for hepatoblastoma, the 2005 revision is intended to be applicable to all primary malignant liver tumours of childhood, including hepatocellular carcinoma and epitheli- oid haemangioendothelioma. The original SIOPEL risk stratification system for hepatoblastoma has already been modified in the protocols for current SIOPEL studies (Table 3). Firstly, tumour rupture or intraperitoneal haemorrhage at the time of diagnosis ( $\mathrm{H} 1$, see below) is now a defining criterion of high risk. Secondly, children with alphafetoprotein levels of $<100 \mu \mathrm{g} / \mathrm{l}$ are also considered to be high risk. The 2005 revision involves no further change in the SIOPEL risk stratification system for hepatoblastoma.

Table 3 Risk stratification in hepatoblastoma for current SIOPEL studies

\begin{tabular}{|c|c|c|}
\hline \multicolumn{2}{|l|}{ High risk } & $\begin{array}{l}\text { Standard risk } \\
\text { All other patients }\end{array}$ \\
\hline $\begin{array}{l}\text { Patients with } \\
\text { any of the } \\
\text { following: }\end{array}$ & $\begin{array}{l}\text { Serum alpha-fetoprotein } \\
<100 \mu \mathrm{g} / 1 \\
\text { PRETEXT IV } \\
\text { Additional PRETEXT } \\
\text { criteria: } \\
\text { E1, E1a, E2, E2a } \\
\text { H1 } \\
\text { M1 (any site) } \\
\text { N1, N2 } \\
\text { P2, P2a } \\
\text { V3, V3a }\end{array}$ & All other patients \\
\hline
\end{tabular}


Fig. 2 PRETEXT I. a The left lateral section (segments 2 and 3 ) is involved. b The right posterior section (segments 6 and 7) is involved
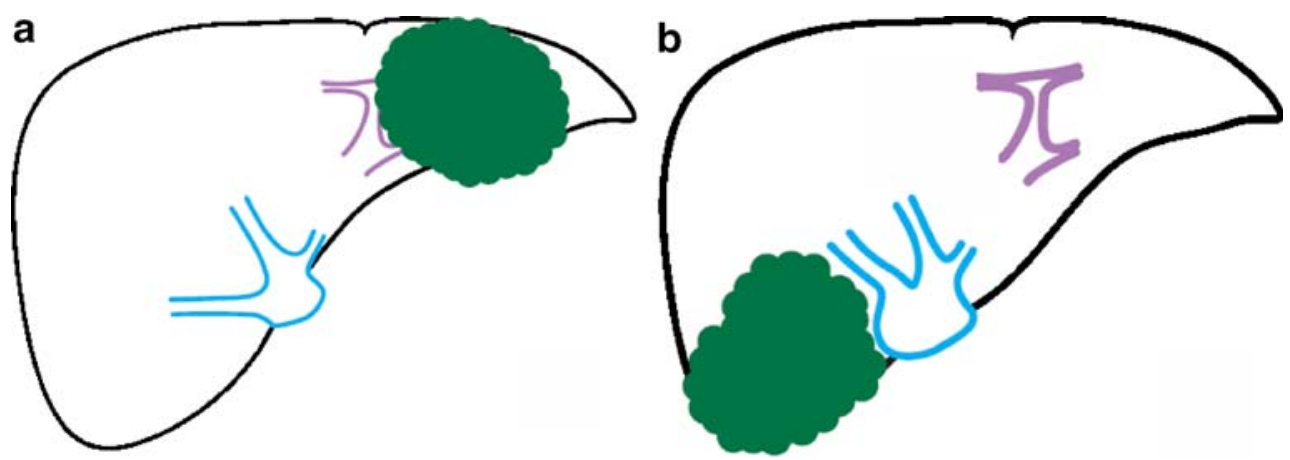

\section{PRETEXT grouping}

The traditional approach to radiological segmentation of the liver, based on the paths of the hepatic veins, is an oversimplification. This is partly due to the variability of hepatic venous anatomy [8-10]. The main problem, however, is the imperfect correlation with segments defined by the branching pattern of the portal veins $[8,11-13]$.
Although the plane of the right hepatic vein reliably separates the right posterior and anterior sections [9], the left hepatic vein runs to the left of the boundary between the left lateral and medial sections, which is best defined by the plane of the fissure of the ligamentum teres and the umbilical portion of the left portal vein (Fig. 1) [14].
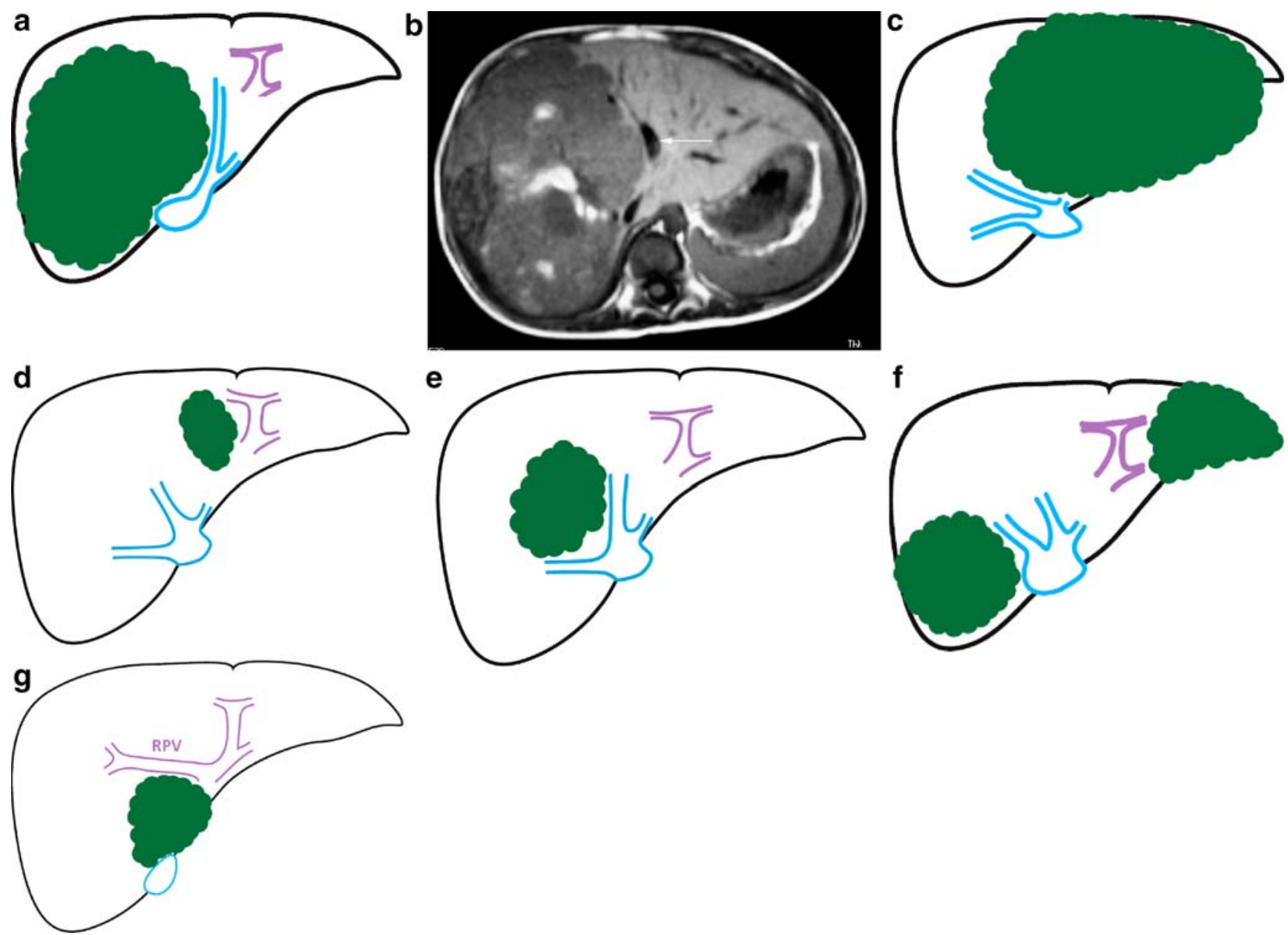

Fig. 3 PRETEXT II. a Tumour involving only the right lobe of the liver. b A transverse T1-weighted MR image of a child with hepatoblastoma shows that the middle hepatic vein (arrow) is displaced but not involved by the tumour. This is the most common type of PRETEXT II tumour. c Tumour involving only the left lobe of the liver. d Tumour involving only the left medial section. e Tumour involving only the right anterior section. f Multifocal tumours involving only the left lateral and right posterior sections. g The tumour is confined to the caudate lobe (PRETEXT II C1, see text; $R P V$ right portal vein) 


\section{PRETEXT I}

This group includes only a small proportion of primary malignant liver tumours of childhood. From the definition of the PRETEXT number, it can be seen that only tumours localized to either the left lateral section or the right posterior section qualify as PRETEXT I (Fig. 2).

\section{PRETEXT II}

Most PRETEXT II tumours are limited to either the right lobe or the left lobe of the liver. Tumours of the left medial or right anterior sections are also PRETEXT II. Multifocal tumours involving only the left lateral and right posterior sections are classified as PRETEXT II; this pattern is very rare. Tumours limited to the caudate lobe were not classifiable under the original PRETEXT system [1]. In the 2005 PRETEXT system these tumours are classified as PRETEXT II (but see also C, below). This is the only change in the PRETEXT numbering system in this revision. There is no change in numbering for tumours involving the caudate lobe and any other part of the liver, which are classified as PRETEXT II (if two or three contiguous sections are free), III (if there are no two contiguous sections free) or IV (if all four sections are involved) (Fig. 3).

\section{PRETEXT III}

The unifocal tumours in this category spare only the left lateral or right posterior section. These tumours are relatively common. In children with hepatoblastoma, great care must be taken to distinguish between invasion and compression of the apparently uninvolved section of the liver, because risk stratification (and/or the need for liver transplantation) may depend on this point. Anterior central liver tumours involve segment 4 and either or both of segments 5 and 8 . Although recent advances in surgical

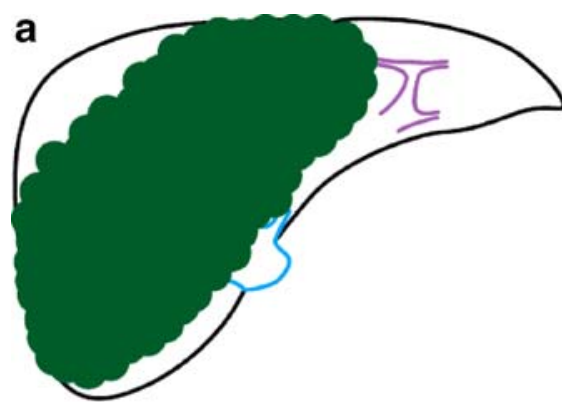

d
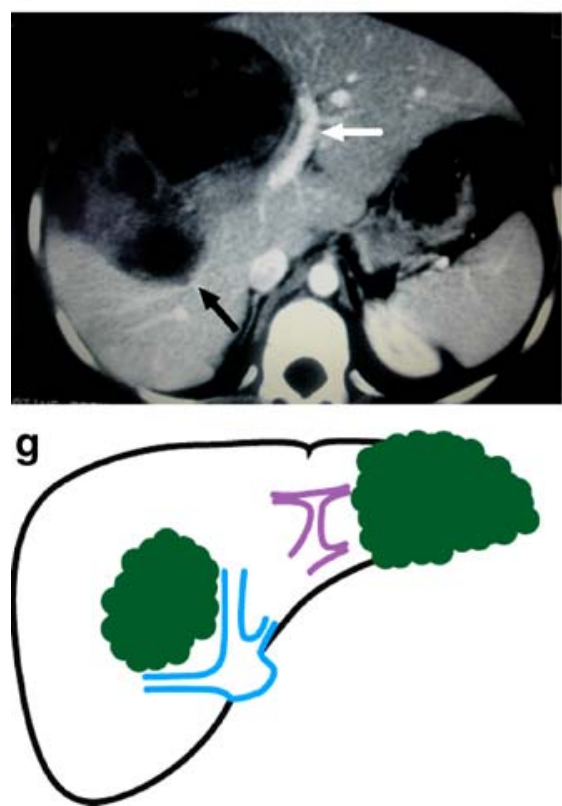

Fig. 4 PRETEXT III. a Extensive tumour sparing only the left lateral section. b Extensive tumour sparing only the right posterior section. c Anterior central liver tumour involving the left medial and right anterior sections. d Contrast-enhanced CT image shows a central liver tumour lying between the left portal vein (white arrow) and the right

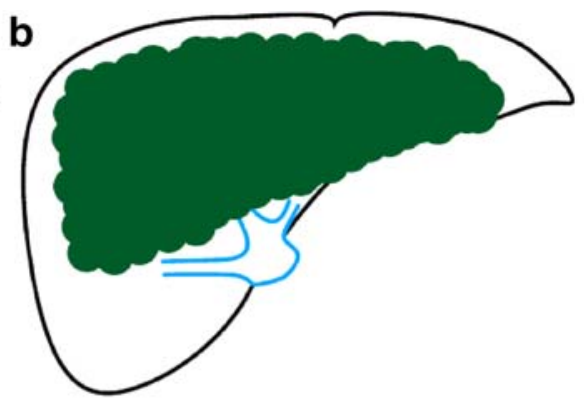

e

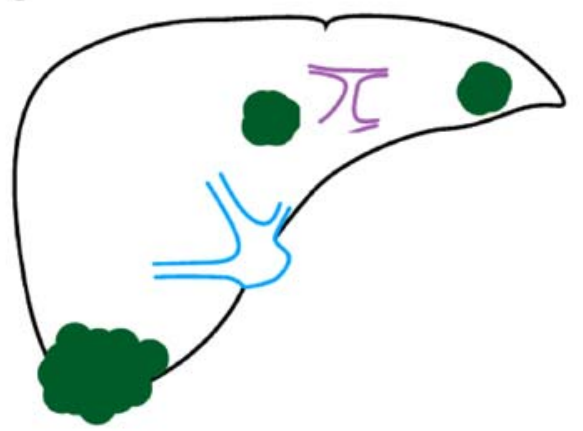

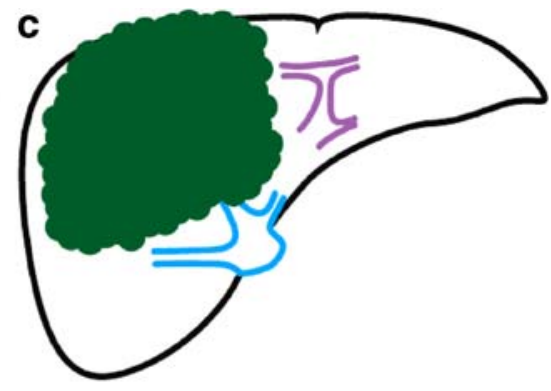

f

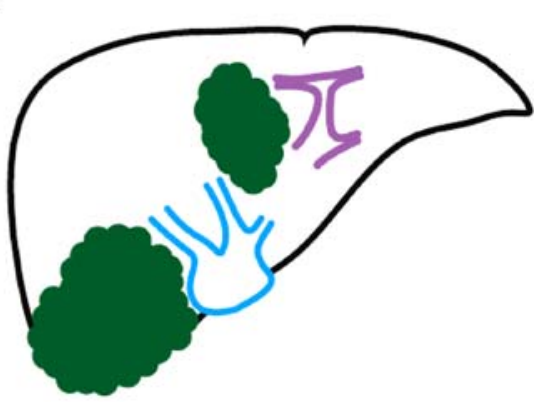

hepatic vein (black arrow). e Multifocal PRETEXT III tumour, sparing the right anterior section. f Multifocal tumours sparing only the left lateral and right anterior sections. $g$ Multifocal tumours sparing only the left medial and right posterior sections 
technique permit resection of these tumours without trisectionectomy [15], classification as PRETEXT III reflects the difficulty of these operations (Fig. 4).

Multifocal PRETEXT III tumours may also spare the right anterior or left medial sections, or two non-contiguous sections. These patterns are rare.

\section{PRETEXT IV}

PRETEXT IV tumours involve all sections of the liver. These tumours are often multifocal. Alternatively, a very large solitary tumour can involve all four sections (Fig. 5).

\section{C: caudate lobe tumours}

The caudate lobe and caudate process (segment 1 or segments 1 and 9, depending on the system of nomenclature) can be resected with either the left or right lobe of the liver [7]. For this reason, segment 1 was not considered in the PRETEXT classification in the original system [2]. Modern surgical techniques have made resection of segment 1 safer, but these operations remain difficult. Involvement of the caudate lobe is, therefore, a potential predictor of poor outcome. If any tumour is present in segment 1 on imaging at diagnosis (Fig. 3g), the patient will be coded as $\mathrm{C}$, irrespective of the PRETEXT group (see above). All other patients should be coded as $\mathrm{C} 0$.

\section{E: extrahepatic abdominal disease}

The assessment of extrahepatic abdominal disease was one of the most confusing aspects of the original PRETEXT system, and clearly needed revision. Originally, there was a requirement for all extrahepatic abdominal spread of tumour $(\mathrm{E}+)$ to be proved by biopsy. Modern imaging techniques are capable, in principle, of identifying extrahepatic abdominal tumour extension in many forms. The frequency and significance of these imaging findings is different for different tumour types, and not all patterns are easily biopsied.

In hepatoblastoma, for example, direct extension of tumour into other abdominal organs is unusual. Tumour extension through the diaphragm (Fig. 6) is uncommon, but can be shown quite convincingly by MRI or CT, and biopsy proof may be impractical. In the 2005 revision, patients with direct extension of tumour through the diaphragm or into other organs can be coded as E1 without biopsy proof.

Pedunculated tumours are considered to be confined to the sections from which they arise, and are not extrahepatic disease.

Peritoneal tumour seeding was originally not included in this category [2]. It probably indicates more advanced abdominal disease than direct extension of the primary tumour. Imaging techniques, especially ultrasonography, can often show even small peritoneal nodules clearly, and the differential diagnosis is very limited. In the 2005 revision, peritoneal nodules will be assumed to be metas-
Fig. 5 PRETEXT IV. a Multifocal PRETEXT IV tumours involve all four sections. b Contrast-enhanced CT image of a patient with PRETEXT IV F1 (see text) hepatoblastoma. c Unifocal PRETEXT IV tumours often have a diffuse growth pattern. d Contrast-enhanced CT image of a patient with diffuse PRETEXT IV hepatoblastoma
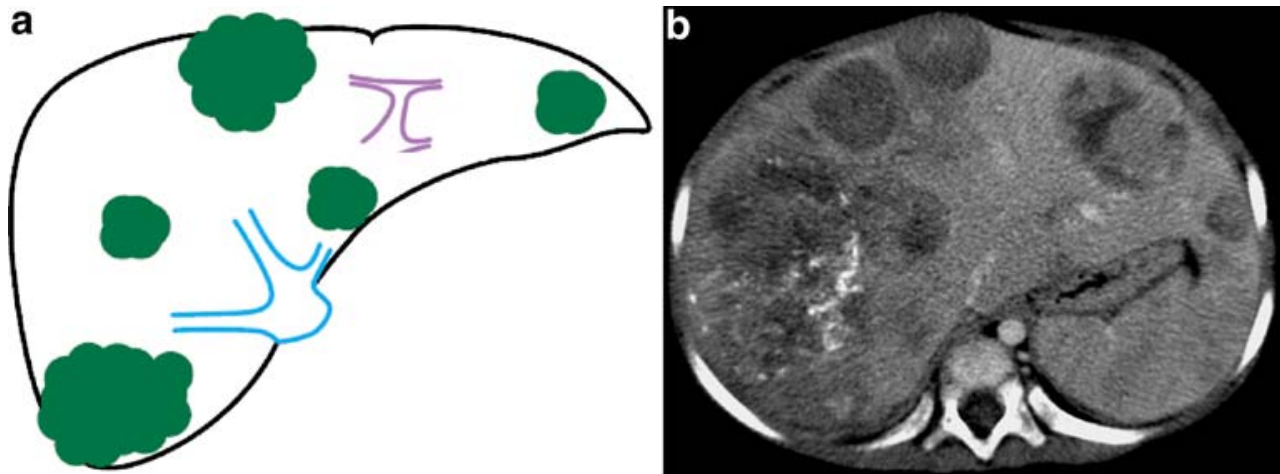

c

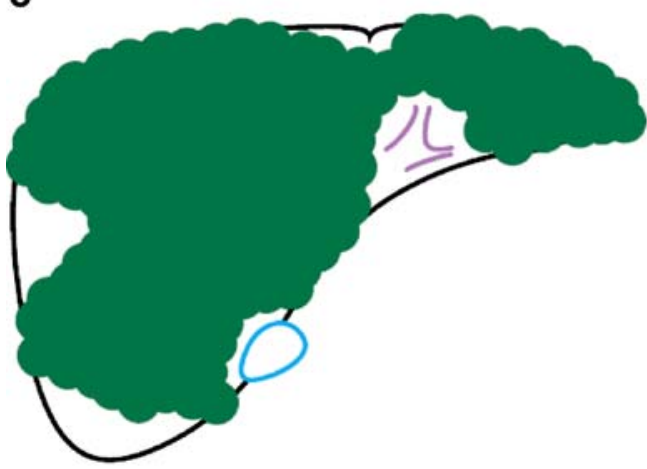


Fig. 6 Extrahepatic abdominal tumour extension. This composite of contrast-enhanced CT images in a patient with hepatoblastoma shows growth of the primary tumour through the diaphragm into the thorax (E1). The 2005 PRETEXT system no longer requires biopsy proof for this form of tumour spread
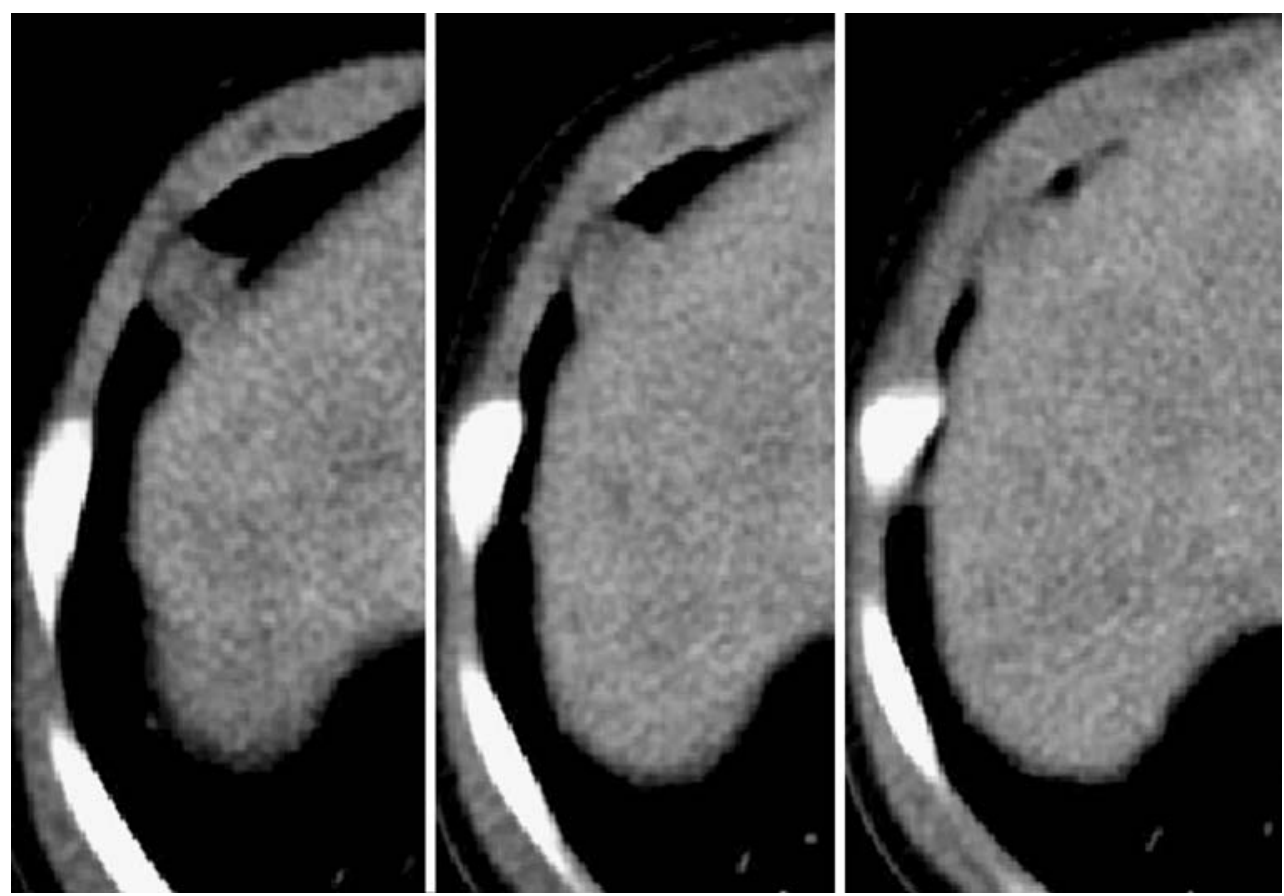

tases, and will be coded as E2. All other patients should be coded as E0.

Ascites is an unusual finding at presentation in hepatoblastoma, but is more common in hepatocellular carcinoma, where it may be an independent predictor of poor prognosis. For this reason, patients with ascites will be coded as E0a, E1a or E2a as appropriate.

Abdominal lymph node metastases, which were previously recorded as $\mathrm{E}+$, are now coded as $\mathrm{N}$ (see below).

\section{F: tumour focality}

In SIOPEL 1, multifocal tumours were identified at the time of diagnosis in $18 \%$ of the patients with hepatoblastoma where this information was available [4]. Univariate analysis showed that the 5-year event-free survival was significantly worse for patients with multifocal tumour $(40 \%)$ than for those with unifocal tumour (72\%) [4]. The independent significance of this finding is unclear, as there is clearly an association between multifocality and advanced PRETEXT number. The German Society of Pediatric Oncology and Hematology reported slightly different results [16]. In its HB89 study, 21\% of patients had multiple well-defined tumours, and these children had a similar disease-free survival (DFS; 87\%) to those with a single tumour $(86 \%)$. However, in $20 \%$ of children the tumour exhibited a diffuse growth pattern (Fig. 5), and these had a significantly worse DFS (21\%) [16]. Unfortunately, a diffuse growth pattern is difficult to define, and despite the promise that this finding shows as a potential risk factor, it was decided not to incorporate it in the 2005 PRETEXT revision.

Patients with one hepatic tumour should be coded as F0. All those with more than one tumour nodule (Figs. 3, 4 and 5), regardless of nodule size or PRETEXT stage, should be coded as F1.

\section{H: tumour rupture or intraperitoneal haemorrhage}

It is not uncommon for hepatoblastoma and hepatocellular carcinoma to present with tumour rupture $[17,18]$. Originally, these patients were not automatically included as high risk in SIOPEL studies, because of the requirement that extrahepatic disease (E) be proved by biopsy. Although the data to prove this are not currently available, it seems intuitively likely that tumour rupture (usually manifesting as intraperitoneal haemorrhage) is a risk factor, and these patients should be coded as H1. Laparotomy or aspiration of peritoneal blood is not necessary for diagnostic purposes if characteristic imaging and clinical findings (such as hypotension and low haematocrit or haemoglobin level) are present. The presence of peritoneal fluid on imaging alone does not imply tumour rupture (but see E above).

Since the opening of the SIOPEL 4 study in September 2004, tumour rupture has become a defining feature of high-risk hepatoblastoma in SIOPEL studies. Patients with no evidence of tumour rupture or haemorrhage, and those with only subcapsular or biopsy-related intraperitoneal bleeding, are coded as H0. 


\section{M: distant metastases}

Patients with distant metastases at diagnosis are coded as M1. In hepatoblastoma, these metastases are predominantly found in the lungs. Although the best imaging modality for the identification of lung metastases is currently CT, the defining characteristics of lung metastases in this context have not been specifically studied. It is believed, however, that factors favouring a diagnosis of metastasis include multiple lesions, a rounded, well-defined contour and a subpleural location. In most parts of the world, a single rounded lung lesion with a diameter of $>5 \mathrm{~mm}$ in a child with a primary liver tumour is very likely to be a metastasis. Patients with these findings on chest CT scans should be classified as M1. Biopsy is not required for staging purposes, because it is uncommon for other lesions to mimic metastases in this clinical context. The protocols of the SIOPEL studies recommend central radiological review if there is any doubt about the presence of lung metastases.

Other metastases are infrequently found at diagnosis in hepatoblastoma, but are more common in hepatocellular carcinoma. The imaging findings of brain metastases are usually characteristic, and biopsy is not required.

Bone scintigraphy is recommended for staging in children with hepatocellular carcinoma, but not hepatoblastoma. Abnormal calcium metabolism is common in children with hepatoblastoma, and may cause abnormal uptake on bone scintigraphy, especially in the ribs [19], whereas bone metastases are rare [20]. Biopsy proof is therefore mandatory for suspected bone metastases in hepatoblastoma, unless the findings of cross-sectional imaging are characteristic and the patient is already in the high-risk category for some other reason, such as the presence of lung metastases.

Bone marrow biopsy is not recommended in children with hepatoblastoma, because bone marrow spread is rare [20]. It is not known whether metastases at different sites have different prognostic implications. For statistical purposes, it is therefore recommended that one or more suffixes be added to M1 to indicate the major sites of metastasis: pulmonary (p), skeletal (s), central nervous system (c), bone marrow (m), and other sites (x). A child with lung, brain, and adrenal metastases would therefore be coded as M1cpx. Patients with no evidence of haematogenous metastatic spread of tumour should be coded as M0.

\section{N: lymph node metastases}

Because porta hepatis (and other abdominal) lymph node metastases are quite unusual in hepatoblastoma, SIOPEL trials have always required this form of tumour spread to be proved by biopsy. In fact, benign enlargement of lymph nodes is probably not uncommon, and the accuracy of positron emission tomography is not known in this context. Because biopsy of equivocal lymph nodes inevitably carries some risk, the SIOPEL committee actively discourages this. Biopsy may, however, be required if there is significant nodal enlargement (for example short axis $>15 \mathrm{~mm}$ ) in a child with no other criteria for high-risk hepatoblastoma.

Lymph node metastases are quite common in hepatocellular carcinoma and fibrolamellar carcinoma, and biopsy proof is not required if the imaging abnormality is unequivocal. An arbitrary threshold short axis diameter of $15 \mathrm{~mm}$ is suggested for this purpose.

Children with no lymph node metastases by these criteria are coded as N0, those with nodal metastases limited to the abdomen (i.e. caudal to the diaphragm and cranial to the inguinal ligament) as N1, and those with extra-abdominal nodal metastases as $\mathrm{N} 2$.

\section{P: portal vein involvement}

Involvement of the main portal vein and/or both major branches has been considered a risk factor in hepatoblastoma, because this has obvious implications for the resectability of the tumour. It is also possible that portal vein invasion detected by imaging is an independent risk factor for tumour recurrence [21]. The original PRETEXT criteria, however, did not specifically define the word "involvement".

It is well recognized that a tumour that abuts or displaces a major portal venous branch at imaging performed at diagnosis (Fig. 7) may shrink away from the vein following preoperative chemotherapy. Imaging evidence of complete obstruction or circumferential encasement (Fig. 7) is therefore required to qualify as portal vein involvement. Failure to identify the portal vein or one of its major branches in either its normal position or its expected displaced location, on good quality images, is strong evidence of obstruction. The other form of involvement, portal vein invasion, is not uncommon, and is often best detected by ultrasound (Fig. 7). Various US signs may be present $[22,23]$, and analogous findings can be seen on CT and MR imaging.

Patients with no imaging evidence of involvement of the main portal vein, its bifurcation, or either of its main branches will be coded as P0. Those who fulfil the original PRETEXT definition of $\mathrm{P}+$ (involvement of the main portal vein, its bifurcation, or both of its main branches), as well as those with "cavernous transformation" of the portal vein will be coded as P2. P2, however, represents very advanced disease. For this reason, the category P1 has been created for patients with evidence of involvement of one major 
Fig. 7 Involvement of the portal and hepatic venous systems. a When the tumour (grey) approaches or abuts the vein (black), there is no venous involvement, even if the vein is partly encased. b Complete obstruction or encasement of the vein is one form of involvement. Obstruction of the inferior vena cava by extrinsic compression, however, does not count as involvement (see text). c Intravascular tumour growth in the portal and/or hepatic venous systems is not uncommon in children with hepatoblastoma or hepatocellular carcinoma.

d Transverse ultrasound image of the right lobe of the liver in a patient with hepatoblastoma. The tumour (white circles) has grown into the right branch of the portal vein $(\mathrm{P} 1 \mathrm{a})$, disrupting the normal "white line" of the vein wall (arrows)
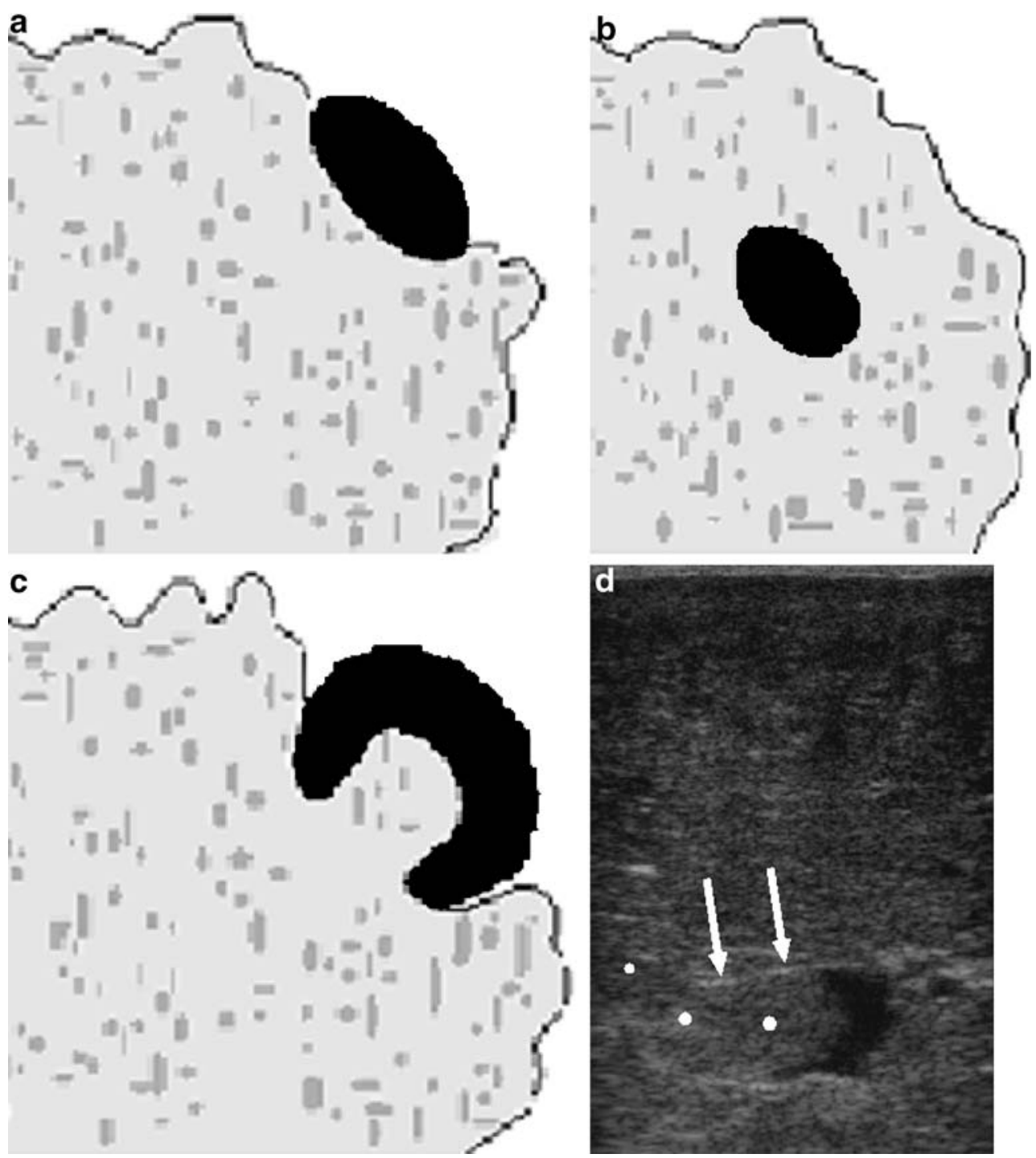

branch of the portal vein. In addition, the detection of portal vein invasion should be marked by the suffix "a" (e.g., $\mathrm{P} 2 \mathrm{a})$.

\section{V: involvement of the IVC and/or hepatic veins}

The same definitions of involvement (venous obstruction, encasement and/or invasion) used for the portal veins apply to the hepatic veins (Fig. 7). A hepatic vein can be assumed to be involved if it cannot be identified at all, and its expected course runs through a large tumour mass. It is important to look carefully for the hepatic veins, preferably with ultrasonography as well as CT and/or MRI, as they may be displaced from their expected position by the tumour. Complete obstruction of the IVC can occur with mass effect alone, without any tumour extension to the vein itself. Inability to visualize the IVC, and the presence of an enlarged azygos vein, are not, therefore, sufficient criteria for involvement. Patients with no imaging evidence of involvement of the hepatic veins or IVC will be coded as V0.

As for the portal vein, the original classification of involvement $(\mathrm{V}+)$ indicated a very advanced level of disease. Intermediate categories have therefore been created. V1 and $\mathrm{V} 2$ indicate involvement of one or two main hepatic veins respectively. V3 indicates involvement of either the IVC or all three of the hepatic veins. In addition, the detection of hepatic vein or IVC invasion should be marked by the suffix "a" (e.g., V2a). The presence of tumour in the right atrium automatically makes a patient V3a.

\section{SIOPEL risk stratification for patients with hepatoblastoma}

The SIOPEL risk stratification for children with hepatoblastoma is essentially unchanged by this revision. Patients 
with any one or more of certain criteria (Table 3) are high risk. All other SIOPEL patients are standard risk.

\section{Presurgical re-evaluation}

Although the timing of surgery will depend on the treatment protocol and the patient's response to therapy, preoperative reimaging is almost always necessary. All of the PRETEXT categories should be reassessed after preoperative chemotherapy, as near as possible to the time of surgery, and recorded as POSTEXT (post-treatment extent of disease). Comparison of surgical findings with POSTEXT will allow prospective assessment of the accuracy of imaging techniques.

Acknowledgements We would like to thank the many radiologists from all over the world who have referred images (some of which have been used in this paper) for a second opinion. PRETEXT staging is heavily dependent on accurate interpretation of high-quality imaging studies. In case of doubt, rapid radiology review is available for patients who may be eligible for SIOPEL studies (see http://www.siopel.org).

\section{References}

1. MacKinlay GA, Pritchard J (1992) A common language for childhood liver tumours. Pediatr Surg Int 7:325-326

2. Pritchard J, Brown J, Shafford E et al (2000) Cisplatin, doxorubicin, and delayed surgery for childhood hepatoblastoma: a successful approach - results of the first prospective study of the International Society of Pediatric Oncology. J Clin Oncol 18:3819-3828

3. Aronson DC, Schnater JM, Staalman CR et al (2005) Predictive value of the pretreatment extent of disease system in hepatoblastoma: results from the International Society of Pediatric Oncology Liver Tumor Study Group SIOPEL-1 study. J Clin Oncol 23:1245-1252

4. Brown J, Perilongo G, Shafford E et al (2000) Pretreatment prognostic factors for children with hepatoblastoma - results from the International Society of Paediatric Oncology (SIOP) study SIOPEL 1. Eur J Cancer 36:1418-1425

5. Perilongo G, Shafford E, Maibach R et al (2004) Risk-adapted treatment for childhood hepatoblastoma. final report of the second study of the International Society of Paediatric Oncology-SIOPEL 2. Eur J Cancer 40:411-421
6. Roebuck DJ, Olsen Ø, Pariente D (2006) Radiological staging in children with hepatoblastoma. Pediatr Radiol 36:176-182

7. Couinaud C (1994) The paracaval segments of the liver. J Hepatobiliary Pancreat Surg 2:145-151

8. Ishibashi Y, Sato TJ, Hirai I et al (2001) Ramification pattern and topographical relationship between the portal and hepatic veins in the left anatomical lobe of the human liver. Okajimas Folia Anat Jpn 78:75-82

9. Murakami G, Hata F (2002) Human liver caudate lobe and liver segment. Anat Sci Int 77:211-224

10. Onishi H, Kawarada Y, Das BC et al (2000) Surgical anatomy of the medial segment (S4) of the liver with special reference to bile ducts and vessels. Hepatogastroenterology 47:143-150

11. Fasel JH, Selle D, Evertsz CJ et al (1998) Segmental anatomy of the liver: poor correlation with CT. Radiology 206:151-156

12. Fischer L, Cardenas C, Thorn M et al (2002) Limits of Couinaud's liver segment classification: a quantitative computer-based three-dimensional analysis. J Comput Assist Tomogr 26:962-967

13. Sales JP, Hannoun L, Sichez JP et al (1984) Surgical anatomy of liver segment IV. Anat Clin 6:295-304

14. Goldsmith NA, Woodburne RT (1957) The surgical anatomy pertaining to liver resection. Surg Gynecol Obstet 105:310-318

15. Ohno Y, Furui J, Kanematsu T (2004) Is a modified central bisegmentectomy a volume-saving operation for pediatric hepatoblastoma? J Pediatr Surg 39:E13-E16

16. Von Schweinitz D, Hecker H, Schmidt-von-Arndt G et al (1997) Prognostic factors and staging systems in childhood hepatoblastoma. Int J Cancer 74:593-599

17. Ishak KG, Glunz PR (1967) Hepatoblastoma and hepatocarcinoma in infancy and childhood. Report of 47 cases. Cancer $20: 396-422$

18. Iida T, Suenaga M, Takeuchi Y et al (2004) Successful resection of a ruptured hepatoblastoma prior to chemotherapy: report of a case. Surg Today 34:710-714

19. Archer D, Babyn P, Gilday D et al (1993) Potentially misleading bone scan findings in patients with hepatoblastoma. Clin Nucl Med 18:1026-1031

20. Exelby PR, Filler RM, Grosfeld JL (1975) Liver tumors in children in the particular reference to hepatoblastoma and hepatocellular carcinoma: American Academy of Pediatrics Surgical Section Survey - 1974. J Pediatr Surg 10:329-337

21. von Schweinitz D, Wischmeyer P, Leuschner I et al (1994) Clinico-pathological criteria with prognostic relevance in hepatoblastoma. Eur J Cancer 30A:1052-1058

22. Sato M, Ishida H, Konno K et al (2000) Liver tumors in children and young patients: sonographic and color Doppler findings. Abdom Imaging 25:596-601

23. Ohtsuka Y, Takahashi H, Ohnuma N et al (1997) Detection of tumor thrombus in children using color Doppler ultrasonography. J Pediatr Surg 32:1507-1510 